\title{
Cambios en la salud sexual de los pacientes obesos tras cirugía bariátrica
}

\author{
Changes in the sexual health of obese patients after bariatric surgery
}

María A. Pomares-Callejón ${ }^{1 *}$, Manuel Ferrer-Márquez² y María J. Solvas-Salmerón ${ }^{3}$

${ }^{1}$ Departamento de Enfermería, Fisioterapia y Medicina, Universidad de Almería; ${ }^{2}$ Servicio de Cirugía General y Digestiva; ${ }^{3}$ Servicio de Urgencias. Complejo Hospitalario Torrecárdenas. Almería, España

\begin{abstract}
Resumen
Introducción: Los objetivos del estudio fueron: 1) evaluar la salud sexual en pacientes con obesidad grave/mórbida candidatos a cirugía bariátrica; y 2) valorar la evolución de la salud sexual tras 12 meses de la cirugía. Método: Estudio descriptivo, prospectivo desde febrero de 2011 hasta junio de 2014. Se valoró la actividad sexual en los hombres a través del cuestionario EVAS-H y la función sexual en la mujer a través de la escala FSM (44 pacientes). Resultados: Durante el estudio basal en los hombres, un $21 \%$ de la muestra presentó disfunción sexual en diferentes dimensiones, mientras que un $43 \%$ presentó problemas de eyaculación precoz. Tras 12 meses de la intervención, se observó un incremento de la actividad sexual global $(p=0.026)$. En torno al $70-89 \%$ de las mujeres, previamente a la cirugía, no presentaban trastorno. En la evolución no se observaron cambios medios relevantes $(p>0.05)$. Conclusiones: Los pacientes con obesidad grave/mórbida candidatos a cirugía bariátrica presentan alteraciones considerables en diversas dimensiones de la salud sexual. Después de 12 meses de seguimiento, la salud sexual parece mejorar en los hombres.
\end{abstract}

PALABRAS CLAVE: Obesidad. Cirugía bariátrica. Salud sexual. Función sexual.

\begin{abstract}
Introduction: The aims of the study were: 1) to assess sexual health patients severe/morbid obesity patients candidates for bariatric surgery; and 2) to assess sexual health evolution after 12 months of surgery. Method: Descriptive, prospective study from February 2011 to June 2014. Sexual activity in men was valued through EVAS-H questionnaire and through FSM scale on women (44 patients). Results: During the basal study in men, a 21\% of the sample showed sexual disfunction in different dimensions, while a $43 \%$ showed problems with premature ejaculation. 12 months after surgery, global sexual activity was improved significantly $(p=0,026)$. Approximately $70-89 \%$ of women presented no disturbance before surgery. No average relevant changes were observed within the evolution $(p>0.05)$. Conclusions: Morbid/severe obesity patients candidates to bariatric surgery, show considerable alterations on diverse sexual health dimensions. After 12 months following bariatric surgery, men's sexual health appears to improve.
\end{abstract}

KEY WORDS: Obesity. Bariatric surgery. Sexual health. Sexual function.

\author{
Correspondencia: \\ *María A. Pomares-Callejón \\ Avda. Mediterráneo, 388, $5^{\circ} 1^{\text {a }} \quad$ Fecha de recepción: 09-03-2018 \\ 04009 Almería, España $\quad$ Fecha de aceptación: 11-04-2018 \\ E-mail: mpc630@ual.es
}

Cir Cir. 2018;86:255-260

Contents available at PubMed www.cirugiaycirujanos.com 


\section{Introducción}

La obesidad representa un proceso patológico y un problema de salud pública en el ser humano. Dicho proceso ha perdurado durante siglos por factores genéticos y ambientales hasta convertirse, actualmente, en una pandemia con consecuencias serias para la salud $^{1,2}$.

La sexualidad constituye una función básica del ser humano que implica factores fisiológicos, emocionales y cognitivos, y que guarda una estrecha relación con el estado de salud y con la calidad de vida del individuo. Del mismo modo que es innegable el impacto potencial que diversos problemas médicos pueden ejercer sobre la salud sexual, también existe un acuerdo generalizado sobre el papel determinante que algunos procesos psicológicos pueden tener en el funcionamiento sexual y en el desarrollo de diferentes disfunciones sexuales ${ }^{3}$. Por tanto, no es de extrañar que la vida sexual de los pacientes con obesidad pueda verse alterada de forma significativa ${ }^{4-7}$. Sin embargo, hasta fechas muy recientes se ha obviado el estudio de las repercusiones de la obesidad sobre la salud sexual, a pesar de que en los últimos años han surgido diversos estudios que abordan esta cuestión ${ }^{4,5,8,9}$.

La disfunción sexual en pacientes obesos es un trastorno común y complejo que puede afectar de manera considerable la calidad de vida de los pacientes. Steffen, et al. ${ }^{10}$ y Bond, et al. ${ }^{11}$ observaron que las mujeres atribuyen principalmente su falta de actividad sexual a estar cansadas, no interesadas o no tener pareja disponible, mientras que los hombres la atribuyen principalmente a dificultades físicas. Diferentes factores se han asociado a la disfunción sexual en estos pacientes, encontrándose como principales los problemas depresivos y la medicación relacionada, los problemas físicos y gran parte de la comorbilidad asociada (enfermedades cardiovasculares, hipertensión arterial, diabetes mellitus) ${ }^{10,12,13}$. Existen algunos trabajos, muy escasos todavía, que han intentado estudiar el efecto de la cirugía bariátrica en la respuesta sexual ${ }^{14-16}$.

Los objetivos de nuestro estudio fueron: 1) evaluar la salud sexual en pacientes con obesidad grave/ mórbida candidatos a cirugía bariátrica; y 2) valorar la evolución de la salud sexual tras la cirugía bariátrica tras 12 meses de la misma.

\section{Método}

\section{Diseño del estudio}

Estudio descriptivo, prospectivo, en el que se analiza la influencia de la cirugía bariátrica sobre la salud sexual de los pacientes obesos graves/mórbidos candidatos a tratamiento quirúrgico, intervenidos de forma consecutiva desde febrero de 2011 hasta junio de 2014.

El proyecto fue aprobado por el comité ético del hospital.

\section{Protocolo para la recogida de datos}

Todos los pacientes fueron evaluados por un equipo multidisciplinario previamente a su tratamiento quirúrgico. Los pacientes fueron intervenidos por la Unidad de Cirugía Bariátrica mediante la realización de bypass gástrico o gastrectomía vertical laparoscópica en función de las características de cada uno y siguiendo los protocolos del centro.

Los criterios de inclusión del estudio fueron pacientes candidatos a cirugía bariátrica con índice de masa corporal (IMC) $\geq 40 \mathrm{~kg} / \mathrm{m}^{2} \mathrm{o} \geq 35 \mathrm{~kg} / \mathrm{m}^{2}$ con comorbilidad asociada; edad comprendida entre 18 y 60 años; obesidad mantenida durante más de 5 años; fracaso de otros tratamientos no quirúrgicos para solucionar la obesidad; y firmar el consentimiento informado para participar en el estudio.

Los pacientes ingresaron el día previo a la intervención, momento en que se les realizó la entrevista personal, se les explicó el estudio y se les ofreció rellenar los cuestionarios EVAS-H (Escala corta para la Evaluación de la Actividad Sexual en el Hombre) y FSM (Función Sexual de la Mujer) ${ }^{17,18}$ (hombres y mujeres, respectivamente), de forma voluntaria y anónima. De la misma manera, tras 12 meses de seguimiento los pacientes volvieron a ser entrevistados en la consulta de cirugía y se les invitó a rellenar el mismo cuestionario.

\section{Variables e instrumento de medida}

EI EVAS-H es un cuestionario diseñado para medir la actividad sexual en hombres. Consta de 10 ítems que miden diferentes dimensiones sexuales. El punto de corte es 38 puntos, y por debajo indicaría la presencia de disfunción sexual. Además, se puede 
corregir por dimensiones en las que puntuaciones $\leq 3$ indicarían la presencia de disfunción ${ }^{17}$. El FSM evalúa la función sexual en las mujeres y se trata de una escala autoadministrada que consta de 14 ítems, tras una pregunta llave. La fiabilidad de dicho cuestionario es de un alfa de Cronbach de 0.83 y evalúa diferentes dimensiones sexuales ${ }^{18}$.

Entre el resto de las variables estudiadas, destacan el peso, el IMC, la existencia de comorbilidad, el tipo de intervención quirúrgica y el porcentaje de exceso de IMC perdido.

\section{Análisis estadístico}

Se realizaron estadísticos descriptivos, que se presentan como media y desviación estándar para las variables cuantitativas, y como número y porcentaje para las variables cualitativas. Se realizó un análisis de la normalidad de las principales variables de estudio, tales como el peso, y las variables de sexualidad mediante histograma y la prueba de Shapiro Wilk.

Para evaluar la salud sexual se realizaron estadísticos descriptivos (para hombres y mujeres por separado, así como para toda la muestra en su conjunto) de los cuestionarios EVAS-H y FSM. La evolución de la salud sexual se evaluó mediante regresión lineal ajustada por la edad, el IMC basal y el estado civil para las variables cuantitativas, así como la prueba de McNemar para las variables cualitativas (al ser muestras pequeñas se calculó el valor de significación mediante la prueba binomial exacta).

\section{Resultados}

Entre febrero de 2011 y junio de 2014 se intervinieron 60 pacientes, a los que se invitó a participar en el estudio. De ellos, 16 no estaban interesados, por lo que se han incluido 44 pacientes (28 mujeres y 16 hombres). La edad media fue de 40 años, y el IMC medio fue de $46.8 \mathrm{~kg} / \mathrm{m}^{2}$. La comorbilidad estuvo presente en todo el estudio, siendo la más frecuente el síndrome de apnea obstructiva del sueño (SAOS), con un porcentaje del $88.9 \%$.

A los 12 meses de la intervención, los pacientes habían experimentado una reducción significativa del IMC, pasando de una media de 46.8 a $31.7 \mathrm{~kg} / \mathrm{m}^{2}$ $(p<0.001)$. En cuanto a la comorbilidad, se produjo una reducción significativa en el SAOS, la dislipidemia y en hipertensión arterial. El síndrome metabólico desapareció y las cifras de pacientes con diabetes mellitus disminuyeron de forma sustancial (Tabla 1).
Tabla 1. Cambios en el peso, el índice de masa corporal y diferentes variables clínicas relacionadas con el riesgo cardiovascular en pacientes con obesidad grave/mórbida tras 12 meses de la intervención de cirugía bariátrica

\begin{tabular}{lcccccc}
\hline & \multicolumn{2}{c}{ Pre } & & \multicolumn{2}{c}{ Post } & p \\
\cline { 2 - 3 } & Media & DE & & Media & DE & \\
\hline Peso, $\mathrm{kg}$ & 133.9 & 22.6 & 88.6 & 15.7 & $<0.001$ \\
IMC $\left(\mathrm{kg} / \mathrm{m}^{2}\right)$ & 46.8 & 7.1 & 31.7 & 6.7 & $<0.001$ \\
Comorbilidad & & $\%$ & & $\%$ & \\
Síndrome metabólico & & 11.1 & & 0.0 & - \\
Hipertensión arterial & & 33.3 & & 7.4 & 0.016 \\
Diabetes mellitus & & 25.9 & & 11.1 & 0.125 \\
Dislipidemia & & 37.0 & & 3.7 & 0.004 \\
SAOS & & 88.9 & & 7.4 & $<0.001$ \\
\hline
\end{tabular}

DE: desviación estándar; IMC: índice de masa corporal; SAOS: síndrome de apnea obstructiva del sueño.

Durante el estudio basal, las puntuaciones medias de la escala EVAS-H no indican, en general, la presencia de disfunción sexual, puesto que el valor medio en la puntuación total del cuestionario fue de 42 puntos. Sin embargo, un $21 \%$ de la muestra estudiada presentó valores inferiores a 38 puntos. Por otro lado, los valores medios por dimensión tampoco indican disfunción (puntuaciones medias $>3$ ), excepto en el caso de la eyaculación precoz, con una puntuación media de 2,67 . En este sentido, un $21 \%$ de la muestra presentó disfunción sexual en las dimensiones de deseo, función eréctil y satisfacción, mientras que un $43 \%$ presentó problemas de eyaculación precoz.

Tras 12 meses de la intervención se observó un incremento en todas las dimensiones de la escala EVAS- $\mathrm{H}$, aunque dicho incremento no fue estadísticamente significativo. La actividad sexual global aumentó significativamente tras la intervención (incremento medio: 8,7 unidades; $p=0.026$ ). Este incremento pudo deberse, en parte, al incremento en la puntuación media de función eréctil que, si bien no fue significativo, mostró una clara tendencia (incremento medio: 4,5 unidades; $p=0.058$ ) (Tabla 2).

Al analizar los cambios en la disfunción sexual global, así como la correspondiente disfunción por dimensiones, se observó que aunque hubo un menor número de pacientes con disfunción sexual en la mayoría de las dimensiones, las diferencias no fueron significativas.

Los resultados relativos a la función sexual de las mujeres mostraron que en las dimensiones deseo, excitación, iniciativa, comunicación y satisfacción 
Tabla 2. Evolución de la media y desviación estándar de las respuestas de la escala EVAS-H al inicio del estudio y a los 12 meses de seguimiento en hombres con obesidad grave/mórbida intervenidos de cirugía bariátrica

\begin{tabular}{lcccccccc}
\hline EVAS-H & \multicolumn{2}{c}{ Pre } & & \multicolumn{2}{c}{ Post } & & p \\
\cline { 2 - 3 } & & & Media & DE & & Media & DE & \\
\hline Deseo (rango: 2-10) & 6.3 & 3.1 & 8.0 & 2.8 & 0.270 \\
Función eréctil (rango: 4-20) & 15.0 & 6.3 & 19.5 & 1.4 & 0.058 \\
Eyaculación precoz (rango: 2-10) & 5.3 & 2.6 & 6.0 & 2.4 & 0.556 \\
Orgasmo (rango: 1-5) & 4.3 & 0.9 & 4.5 & 1.1 & 0.619 \\
Satisfacción (rango: 2-10) & 6.8 & 2.4 & 7.9 & 2.1 & 0.343 \\
Dolor (rango: 1-5) & 4.6 & 0.7 & 4.9 & 0.4 & 0.423 \\
Puntuación total (0-60) & 42.1 & 7.7 & 50.8 & 5.9 & 0.026 \\
\hline DE: desviación estándar. & & & & &
\end{tabular}

(actividad sexual y sexual general), en torno al 70$89 \%$ de las mujeres no presentaban trastorno. Además, un $31 \%$ declaró tener actividad sexual una o dos veces por semana y un $31 \%$ declaró tener actividad sexual entre cinco y ocho veces por semana en las últimas 4 semanas. Sin embargo, se pudo observar la existencia de un importante trastorno en la lubricación en un $15 \%$ de la muestra, un trastorno moderado o importante en el orgasmo en un 4 y un $12 \%$, respectivamente, un alto porcentaje del $58 \%$ de trastorno moderado en problemas con la penetración vaginal, y un $73 \%$ de trastorno importante en ansiedad anticipatoria.

La evolución de la puntuación media de las diferentes dimensiones de la función sexual de la mujer se presenta en la tabla 3 . No se observaron cambios medios relevantes en ninguna dimensión de la función sexual $(p>0.05)$.

\section{Discusión}

La obesidad se ha convertido en la epidemia del siglo xxi, con repercusiones importantes para la salud del individuo que la padece. Como parte de ella, la salud sexual y los diferentes dominios de la sexualidad pueden verse alterados en este tipo de pacientes. En el presente estudio se observó un $21 \%$ de disfunción eréctil en los hombres, lo que concuerda con estudios previos ${ }^{6,19-22}$ que muestran prevalencias de disfunción eréctil de un $12-36 \%$. Moreira, et al. ${ }^{20}$, en el año 2000, en una muestra de 342 hombres, detectaron disfunción eréctil en un $12 \%$. Sin embargo, tanto Fung, et al. ${ }^{19}$ como Cheng, et al. ${ }^{21}$ encontraron porcentajes más altos de disfunción eréctil (36 y 36,7\%,
Tabla 3. Evolución media de las respuestas del cuestionario FSM al inicio del estudio y a los 12 meses de seguimiento en mujeres con obesidad grave/mórbida intervenidas de cirugía bariátrica

\begin{tabular}{|c|c|c|c|c|c|}
\hline \multirow[t]{2}{*}{ FSM } & \multicolumn{2}{|c|}{ Pre } & \multicolumn{2}{|c|}{ Post } & \multirow[t]{2}{*}{$p$} \\
\hline & Media & $\mathrm{DE}$ & Media & $\mathrm{DE}$ & \\
\hline Deseo (rango: 1-15) & 10.6 & 2.5 & 10.8 & 3.4 & 0.850 \\
\hline $\begin{array}{l}\text { Excitación } \\
\text { (rango: 1-15) }\end{array}$ & 11.4 & 3.3 & 11.3 & 3.2 & 0.721 \\
\hline $\begin{array}{l}\text { Lubricación } \\
\text { (rango: 1-5) }\end{array}$ & 3.8 & 1.4 & 3.6 & 1.6 & 0.893 \\
\hline $\begin{array}{l}\text { Orgasmo } \\
\text { (rango: } 1-5)\end{array}$ & 4.6 & 1.0 & 4.5 & 1.1 & 0.617 \\
\hline $\begin{array}{l}\text { Problemas } \\
\text { penetración } \\
\text { vaginal } \\
\text { (rango: 1-15) }\end{array}$ & 7.1 & 1.8 & 7.9 & 1.9 & 0.230 \\
\hline $\begin{array}{l}\text { Ansiedad } \\
\text { anticipatoria } \\
\text { (rango: } 1-5 \text { ) }\end{array}$ & 1.5 & 1.2 & 1.4 & 0.9 & 0.848 \\
\hline $\begin{array}{l}\text { Iniciativa } \\
\text { sexual } \\
\text { (rango: 1-5) }\end{array}$ & 3.2 & 1.2 & 2.9 & 0.8 & 0.696 \\
\hline $\begin{array}{l}\text { Comunicación } \\
\text { sexual (rango: 1-5) }\end{array}$ & 3.9 & 1.4 & 4.3 & 1.1 & 0.495 \\
\hline $\begin{array}{l}\text { Satisfacción } \\
\text { con la actividad } \\
\text { sexual (rango: 1-10) }\end{array}$ & 9.3 & 1.3 & 9.1 & 2.1 & 0.688 \\
\hline $\begin{array}{l}\text { Satisfacción sexual } \\
\text { general } \\
\text { (rango: 1-10) }\end{array}$ & 4.1 & 0.7 & 4.3 & 1.2 & 0.854 \\
\hline
\end{tabular}

DE: desviación estándar.

respectivamente), con tamaños muestrales amplios y usando el cuestionario Índice Internacional de Función Eréctil (IIEF).

Por otra parte, cabe destacar el alto porcentaje de hombres con eyaculación precoz al inicio del estudio, en línea con los hallazgos de Dallal, et al. ${ }^{23}$, quienes usando el cuestionario Brief Sexual Function Inventory (BSFI) encontraron puntuaciones en función eyaculatoria significativamente inferiores a las de la población general. Estos resultados destacan la importancia de evaluar otras dimensiones de la sexualidad de los hombres con obesidad mórbida, además de la función eréctil.

Respecto a la función sexual de la mujer, cabe destacar los altos porcentajes de trastorno moderado en penetración vaginal $(58 \%)$, posiblemente influenciados por el $15 \%$ observado de problemas importantes en lubricación, así como el alto porcentaje de mujeres con trastorno grave en cuanto a ansiedad 
anticipatoria (73\%). Los porcentajes respecto a otras alteraciones de la sexualidad femenina, como trastornos del deseo, excitación y orgasmo, se situaron en torno al $15 \%$, aglutinando los porcentajes de trastorno moderado y grave, un porcentaje algo menor que en la mayoría de los estudios previos.

Janik, et al. ${ }^{24}$ publicaron en 2014 un estudio transversal en el que una serie de 153 mujeres respondieron a los cuestionarios Female Sexual Function Index (FSFI) y Sexual Quality of Life-Female (SQoL-F). Los resultados obtenidos en el preoperatorio según el FSFI afirmaron que 10 de cada 20 mujeres $(50 \%)$ presentaban disfunción sexual. Concluyeron que la obesidad reduce la calidad de vida, incluyendo la calidad sexual, puesto que causa problemas específicos sexuales, incluyendo un deseo sexual disminuido y una menor frecuencia de encuentros sexuales 24,25 .

Jiménez, et al. ${ }^{26}$, en 2014, utilizaron el cuestionario FSM en 50 mujeres candidatas a cirugía bariátrica. Sus resultados con respecto a trastornos de lubricación son similares a los obtenidos en nuestro estudio $(12,5 \%)$. Con respecto a los problemas en la penetración vaginal y en ansiedad anticipatoria, sus porcentajes fueron mucho más bajos (12,5 y $6,25 \%$, respectivamente).

Tras 12 meses de seguimiento se observó una mejora de la actividad sexual en los hombres, a juzgar por el incremento de casi 9 unidades (15\%) en la puntuación global de la escala EVAS-H. Los resultados mostraron que este incremento se debió en parte a la mayor puntuación en función eréctil (4,5 unidades de diferencia respecto al inicio del estudio), aunque el resto de las dimensiones del cuestionario también aumentaron su puntuación (pero de forma no significativa). Lobató, et al. ${ }^{27}$, en su estudio cualitativo/cuantitativo de 2011, tras un periodo de seguimiento de 12 meses después de la cirugía con bypass gástrico, demostraron una mejoría en los dominios deseo, función eréctil y orgasmo, lo que está en línea con los presentes resultados. En 2012, Goitein, et al. ${ }^{25}$ llevaron a cabo un estudio en mujeres y hombres que presentaban obesidad mórbida y que fueron intervenidos mediante gastrectomía vertical laparoscópica y bypass gástrico. Se utilizaron los cuestionarios FSFI en mujeres y BSFI en hombres, antes y 6-7 meses después de la cirugía. Tras la cirugía, el BSFI aumentó de 40.2 a 43.9 , pero no se alcanzó significación estadística. Sin embargo, aun no encontrando esa diferencia, hubo una mejora en las dimensiones satisfacción general, deseo y erección, comparable a la establecida en nuestros resultados, los cuales son similares excepto en el hecho de que nosotros sí encontramos diferencias significativas en la puntuación global de la actividad sexual entre antes y después de la intervención.

Estos resultados, en conjunto, indican que la actividad sexual en los hombres puede mejorar de manera considerable tras la cirugía bariátrica, lo que tiene gran importancia clínica y de salud pública, y refuerza los beneficios de dicha cirugía como primera línea de tratamiento de la obesidad grave/mórbida. Sin embargo, se necesita más investigación para determinar los efectos a largo plazo (2-5 años), así como las dimensiones concretas en las que particularmente se producen las mejoras.

Las diferentes dimensiones de la función sexual de la mujer, a juzgar por los resultados del seguimiento utilizando el cuestionario FSM, no mostraron diferencias significativas tras la cirugía. Assimakopoulos, et al. ${ }^{8}$, en 2010, en una muestra de 59 mujeres, a través del FSFI concluyeron que tras 1 año de la cirugía todos los dominios sexuales en las mujeres con obesidad mejoraban, excepto la ansiedad y el orgasmo. Así mismo, en el análisis estadístico tanto el IMC como las dimensiones deseo, excitación, lubricación, satisfacción y función sexual sufrieron una mejora significativa; resultados similares a los de Janik et al. ${ }^{24}$, quienes revelaron que a los 12-18 meses de la cirugía los resultados del FSFI eran más altos en lo que concierne a los dominios deseo y excitación tras la operación.

Así mismo, Bond, et al. ${ }^{11}$, tras 6 meses de la intervención quirúrgica, encuentran que el $22 \%$ seguían presentando disfunción sexual, tal como ocurre en nuestros resultados, aunque tuvieron una mejora en todos los dominios excepto en deseo y lubricación.

El estudio presenta limitaciones que deben ser señaladas. En primer lugar, el tamaño muestral fue relativamente pequeño, aunque bien es cierto que la mayor parte de los estudios sobre sexualidad en esta población no son de gran tamaño muestral 19,11,24,26-29. Por otro lado, se incluyeron pacientes intervenidos mediante dos técnicas quirúrgicas diferentes, cuyos resultados ponderales tienden a variar, lo cual podría haber afectado al estudio de la asociación entre pérdida de peso y cambios en la salud sexual en el seguimiento.

Como conclusión, los pacientes con obesidad grave/mórbida candidatos a cirugía bariátrica presentan alteraciones considerables en diversas dimensiones de la salud sexual. Después de 12 meses de 
seguimiento tras la cirugía bariátrica, hombres y mujeres tuvieron resultados diferenciados. Por un lado, la actividad sexual global de los hombres mejoró de manera significativa, posiblemente promovido por una mejora en la función eréctil, lo cual requiere futura investigación; y por otro lado, la función sexual en las mujeres no cambió tras 12 meses de seguimiento.

\section{Responsabilidades éticas}

Protección de personas y animales. Los autores declaran que para esta investigación no se han realizado experimentos en seres humanos ni en animales.

Confidencialidad de los datos. Los autores declaran que han seguido los protocolos de su centro de trabajo sobre la publicación de datos de pacientes.

Derecho a la privacidad y consentimiento informado. Los autores han obtenido el consentimiento informado de los pacientes o sujetos referidos en el artículo. Este documento obra en poder del autor de correspondencia.

\section{Conflicto de intereses}

Los autores declaran que no tienen conflicto de intereses.

\section{Bibliografía}

1. González E. Obesity: etiologic and pathophysiological analysis. Endocrinol Nutr. 2013;60:17-24. (Consultado el 10 de enero de 2018.) Disponible en http://www.sciencedirect.com/science/article/pii/S2173509313000081.

2. Cuesta AL, Sabán J. La obesidad como entidad pluripatológica. Epidemiología. Sistemas neurohormonales. En: Sabán J, editor. Madrid: Díaz de Santos; 2012. p. 246-52.

3. Carrobles J, Gámez M, Almendro C. Funcionamiento sexual, satisfacción sexual y bienestar psicológico y subjetivo en una muestra de mujeres españolas. An Psicol. 2011;27:27-34.

4. Kolotkin RL, Zunker C, Østbye T. Sexual functioning and obesity: a review. Obesity. 2012;20:2325-33.

5. Larsen SH, Wagner G, Heitmann BL. Sexual function and obesity. Int J Obes. 2007;31:1189-98.

6. Bajos N, Wellings K, Laborde C, Moreau C. Sexuality and obesity, a gender perspective: results from French national random probability survey of sexual behaviours. BMJ. 2010;340:c2573.

7. Carr D, Murphy LF, Batson HD, Springer KW. Bigger is not always better: the effect of obesity on sexual satisfaction and behavior of adult men in the United States. Men Masc. 2013;16:452-77.
8. Assimakopoulos K, Karaivazoglou K, Panayiotopoulos S, Hyphantis T, Iconomou G, Kalfarentzos F. Bariatric surgery is associated with reduced depressive symptoms and better sexual function in obese female patients: a one-year follow-up study. Obes Surg. 2011;21:362-6.

9. Efthymiou V, Hyphantis T, Karaivazoglou K, Gourzis P, Alexandrides TK, Kalfarentzos $F$, et al. The effect of bariatric surgery on patient HRQOL and sexual health during a 1-year postoperative period. Obes Surg. 2015;25:310-8.

10. Steffen KJ, King WC, White GE, Subak LL, Mitchell JE, Courcoulas AP, et al. Sexual functioning of men and women with severe obesity before bariatric surgery. Surg Obes Relat Dis. 2017;13:334-43.

11. Bond DS, Wing RR, Vithiananthan S, Sax HC, Roye GD, Ryder BA, et al. Significant resolution of female sexual dysfunction after bariatric surgery. Surg Obes Relat Dis. 2011;7:1-7.

12. Assimakopoulos K, Panayiotopoulos S, Iconomou G, Karaivazoglou K, Matzaroglou C, Vagenas $\mathrm{K}$, et al. Assessing sexual function in obese women preparing for bariatric surgery. Obes Surg. 2006;16:1087-91.

13. Trischitta V. Relationship between obesity-related metabolic abnormalities and sexual function. J Endocrinol Invest. 2003;26:62-4.

14. Camps MA, Zervos E, Goode S, Rosemurgy AS. Impact of bariatric surgery on body image perception and sexuality in morbidly obese patients and their partners. Obes Surg. 1996;6:356-60.

15. Kinzl JF, Trefalt E, Fiala M, Hotter A, Biebl W, Aigner F. Partnership, sexuality, and sexual disorders in morbidly obese women: consequences of weight loss after gastric banding. Obes Surg. 2001;11:455-8.

16. Sarwer DB, Lavery M, Spitzer JC. A review of the relationships between extreme obesity, quality of life, and sexual function. Obes Surg. 2012;22:668-76

17. Águila $\mathrm{E}$, Sánchez L, Daza MT, La Calle P, Fernández I. Disfunciones sexuales en pacientes con DCA: estudio exploratorio descriptivo con el cuestionario EVAS-M y EVAS-H. Congreso Español de Sexología, V Encuentro Iberoamericano de Profesionales de la Sexología; 2010.

18. Sánchez F, Pérez M, Borrás JJ, Gómez O, Aznar J, Caballero A. Diseño y validación del Cuestionario de Función Sexual de la Mujer (FSM). Aten Primaria. 2004;34:286-92.

19. Fung MM, Bettencourt R, Barret-Connor E. Heart disease risk factors predict erectile dysfunction 25 years later: the Rancho Bernardo Study. J Am Coll Cardiol. 2004;43:1405-11.

20. Moreira ED, Bestane WJ, Bartolo EB, Fittipladi JA. Prevalence and determinants of erectile dysfunction in Santos, southeastern Brazil. Sao Paulo Med J. 2002;120:49-54.

21. Cheng JY, Ng EM. Body mass index, physical activity and erectile dysfunction: an U-shaped relationship from population-based study. Int $J$ Obes. 2007;31:1571-8.

22. Han TS, Tajar A, O'Neill TW, Jiang M, Bartfai G, Boonen S, et al. Impaired quality of life and sexual function in overweight and obese men: the European Male Ageing Study. Eur J Endocrinol. 2011;164:1003-11.

23. Dallal RM, Chernoff A, O'Leary MP, Smith JA, Braverman JD, Quebbemann BB. Sexual dysfunction is common in the morbidly obese male and improves after gastric bypass surgery. J Am Coll Surg. 2008;207:859-64.

24. Janik MR, Bielecka I, Paśnik K, Kwiatkowski A, Podgórska L. Female sexual function before and after bariatric surgery: a cross-sectional study and review of literature. Obes Surg. 2015;25:1511-7.

25. Goitein D, Zendel A, Segev L, Feigin A, Zippel D. Bariatric surgery improves sexual function in obese patients. IMAJ. 2015;17:616-9.

26. Jiménez C, Macías P, Pérez R, Cano A, Domínguez E. Impacto de la cirugía bariátrica en la función sexual de la mujer. BMI. 2014;4.5.8:5905. (Consultado el 20 de enero de 2018.) Disponible en: http://bmilatina. com/index.php/bmi/article/view/239

27. Lobató ML, Boccara MA, Germano D, Roberto P. Bariatric surgery: impact on sexuality of the obese person. Rev Col Bras Cir. 2014:41:412-20.

28. Esposito K, Ciotola M, Giugliano F, Bisogni C, Schisano B, Autorino R, et al. Association of body weight with sexual function in women. Int $J$ Impot Res. 2007;19:353-7.

29. Groutz A, Gordon D, Schachter P, Amir H, Shimonov M. Effects of bariatric surgery on male lower urinary tract symptoms and sexual function. Neurourol Urodyn. 2017;36:636-9. 